Ciencia y Educación, Vol. 5, No. 3, septiembre-diciembre, 2021

ISSN (impreso): 2613-8794・ISSN (en línea): 2613-8808

DOI: https://doi.org/10.22206/cyed.2021.v5i3.pp7-18

\title{
La mediación parental en la pandemia: dispositivos, género y distribución del trabajo
}

\author{
Parental mediation in the pandemic: Devices, gender \\ and labour division
}

Carolina Duek a ORCID: 0000-0002-3103-0363

Marina Moguillansky ${ }^{b}$ ORCID: 0000-0002-8873-1136

Recibido: 31/03/21 - Aprobado: 28/06/21

Cómo citar: Duek, C., \& Moguillansky, M. (2021). La mediación parental en la pandemia: dispositivos, género y distribución del trabajo. Ciencia y Educación, 5(3), 7-18. Doi: https://doi.org/10.22206/cyed.2021.v5i3.pp7-18

\section{Resumen}

Este artículo se propone analizar cómo se recrean las reglas familiares de uso de tecnologías digitales durante el año 2020 en el marco de la pandemia en Argentina. El enfoque es sociológico y el estudio fue cualitativo. Con muestreo teórico se realizaron veinte entrevistas estructuradas a padres o madres de niños y niñas de seis a nueve años de edad, de escuelas públicas y privadas del área metropolitana de Buenos Aires. Se estudiaron las mediaciones parentales, entendidas como las estrategias de gestión del vínculo de los niños con las tecnologías digitales, con relación al uso de dispositivos, la distribución de tareas domésticas y con el género. Se plantea que se han modificado las modalidades de mediación en diálogo con resultados de investigaciones previas que se exponen y comparan. La pandemia modificó la forma de pensar y gestionar los permisos y los límites de la conectividad de niños y niñas.

Palabras clave: pandemia, familia, género, infancia, tecnología.

\begin{abstract}
This article intends to analyze how family regulations on the use of digital technologies were deployed during 2020 in the context of the pandemic in Argentina. This sociological study has a qualitative design. Using theoretical sampling, the field work consisted of twenty structured interviews with parents of 6 to 9 year-old children, from public and private schools in the Metropolitan Area of Buenos Aires. Parental mediations, defined as strategies deployed to manage the relationship of the youngest with digital technologies, are analyzed in relation to the devices use, the domestic tasks distribution and the gender. The parental mediation modalities have been modified in contrast to the results of previous investigations that are exposed and compared in the article. The pandemic changed the ways of thinking and managing the permissions and limits regarding children's online activities.
\end{abstract}

Keywords: Pandemic, family, gender, childhood, technology.

\footnotetext{
a Universidad de Buenos Aires, Argentina. Correo-e: duekcarolina@gmail.com

b Universidad de Buenos Aires, Argentina. Correo-e: mmoguillansky@gmail.com
} 


\section{Introducción}

El año 2020 fue desafiante para todos los ciudadanos y las ciudadanas del mundo: la pandemia por el SARS-CoV-2 exigió una reestructuración de la vida cotidiana, el trabajo, la educación y la sociabilidad. El confinamiento, el cierre de comercios y de las escuelas fueron medidas frecuentes en casi todo el mundo. El virus exigía aislamiento y reclusión para evitar su propagación y la saturación del sistema sanitario. En la Argentina, la resolución 180/2020 estableció que desde el 16 de marzo de 2020 se suspendía el dictado de clases presenciales en todos los niveles educativos por un período de 14 días $^{\mathrm{a}}$. Así, desde el 20 de marzo los comercios, las instituciones, las actividades y el transporte considerados no esenciales fueron cerrados. La circulación solo estaba permitida para el personal de salud y otras actividades esenciales, tales como supermercados y farmacias, que funcionaban en horarios restringidos. En consecuencia, la vida cotidiana se transformó de manera drástica durante todo el año 2020.

El cierre de las escuelas en Argentina fue objeto de polémicas y de diversos estudios cruzados sobre las consecuencias que esta medida tiene sobre las infancias (Baratta, 2021; Duek, 2021). Las escuelas estuvieron cerradas (algunas se usaron como centros de testeo de COVID-19) hasta febrero de 2021, con una apertura puntual en diciembre para un encuentro de socialización y vinculación de los alumnos. El 2020 fue un año de educación a distancia, de nuevas destrezas y formas de vincularse. En este contexto, se ha planteado como objetivo analizar las mediaciones parentales del uso de tecnologías digitales para identificar su gestión y seguimiento durante la pandemia, junto con las dinámicas y las posibilidades de uso durante el ciclo lectivo no presencial 2020.

Al respecto, es importante saber que, aunque Argentina es actualmente el país latinoamericano con mayor tasa de conectividad a internet en los hogares
(67\%), aún cuenta con brechas de acceso y con sectores que no cuentan con dispositivos ni conectividad (Becerra, 2021). Con una población de 44,27 millones de habitantes (INDEC, 2017), la conectividad a redes $4 \mathrm{G}$ pasó de 5 millones de usuarios en 2015, a 29 en diciembre de 2018, y los dos principales problemas para quienes acceden a la conexión son, según informes oficiales, las elevadas tarifas y la velocidad de conexión (Giudici, 2018; Becerra, 2021). El $80,1 \%$ de la población tenía en 2017 acceso a conexión a internet con un promedio de 4 horas y 11 minutos al día (SINCA, 2017); el mismo informe reportó que 6 de cada 10 habitantes contaba con un teléfono móvil individual. El aumento de la conectividad y del acceso a Internet durante el año 2020 no se ha publicado aún, pero se cuenta con datos preliminares en el informe reciente de Becerra (2021).

La transformación de la educación presencial a no presencial, con las múltiples modalidades que asumió, junto con los datos de conectividad y de tiempos de uso de los dispositivos, componen un contexto que impone nuevos desafíos y nuevas preguntas para la investigación en el marco de la pandemia.

El presente artículo se divide en tres secciones. En la primera se describe el diseño metodológico basado en un corpus de veinte entrevistas realizadas a padres y madres con hijos de entre seis y nueve años de edad. En la segunda sección se reconstruyen las discusiones teóricas acerca de la paternidad digital y se propone una caracterización de las formas de mediación parental sobre los usos de tecnologías en la infancia antes de la pandemia. En la tercera sección se analizan los cambios en las reglas de uso de dispositivos electrónicos, a partir de los testimonios de las familias entrevistadas. En síntesis, el núcleo analítico de este trabajo consiste en una revisión de las discusiones sobre el concepto de mediación parental y en su revisión en el contexto de la pandemia y de las transformaciones que implicó para las familias con niños en edad escolar el ciclo lectivo no presencial durante el año 2020.

${ }^{a}$ El decreto completo se encuentra en http://servicios.infoleg.gob.ar/infolegInternet/verNorma.do?id=335423 (último acceso: 15 de junio de 2021).

8 - Ciencia y Educación 2021; 5(3, septiembre-diciembre): 7-18 • Artículos de investigación 


\section{Diseño teórico-metodológico}

Este trabajo discute los cambios en las mediaciones parentales con respecto a los usos de pantallas en niños y nińas. Para esto, el estudio realizado utilizó datos de investigaciones previas (propias y ajenas) para caracterizar las formas de mediación parental previas a la pandemia y datos nuevos para analizar las transformaciones actuales.

Entre los años 2016 y 2019 se desarrolló una extensa investigación sociológica de carácter cualitativo en diversas regiones de la Argentina, que contó con 85 entrevistas y 22 grupos focales (Proyecto PIO CONICET FLACSO N009, dirigido por el Dr. Benítez Larghi, 2016-2019). En ese trabajo de campo se indagaron los usos de las tecnologías digitales y el carácter configurador del género con respecto a las prácticas y formas de uso de pantallas en las infancias. Los datos fueron recabados en grupos focales en escuelas públicas y privadas, complementadas luego con entrevistas individuales con los niños, las niñas, sus padres o madres y los docentes. Los datos fueron codificados y analizados a través del método de comparación constante utilizando el software Atlas ti. Algunos de los resultados del proyecto fueron publicados en Benítez Larghi, Duek \& Moguillansky, 2017 y Duek \& Moguillansky, 2020.

A comienzos de 2021 se realizó un nuevo trabajo de campo destinado a analizar las transformaciones de las mediaciones parentales de las tecnologías en la pandemia. Siguiendo los principios del muestreo teórico, se hicieron veinte entrevistas estructuradas a padres/madres de niños y niñas de seis a nueve años de edad, que asisten a escuelas públicas y privadas en el área metropolitana de Buenos Aires. Las respuestas se solicitaron a través de una aplicación de mensajería instantánea, en mensajes de texto y de voz. Esta metodología permitió un trabajo de campo cualitativo a la distancia, con flexibilidad horaria, y produjo un proceso reflexivo novedoso, brindando a los respondientes la posibilidad de tomarse un tiempo para pensar en las preguntas y luego responder. A su vez, esta dinámica permite las repreguntas y los esclarecimientos o reformulaciones.
Los ejes de las entrevistas fueron la vida cotidiana familiar en pandemia, las reglas de uso de pantallas para las infancias, el acompañamiento escolar y la rutina educativa a la distancia, con sus dificultades y ventajas, en el contexto de una "nueva normalidad" durante el año 2020.

Para el análisis de los datos se propusieron dos hipótesis. La primera hipótesis se refiere a la regulación del tiempo y las formas de exposición a las pantallas por parte de niños y niñas, y sostiene que los hogares donde había una alta regulación del uso antes de la pandemia flexibilizaron estos límites. Por el contrario, en las familias en las que había una baja regulación de uso de pantallas, la pandemia dio lugar a nuevas formas de mediación parental que limitaron o regularon los usos y el tiempo de exposición a pantallas. La segunda hipótesis se vincula con el género y la distribución de las tareas de mediación parental de las tecnologías y afirma que la mediación parental, que antes de la pandemia estaba a cargo de las mujeres (Duek \& Moguillansky, 2020), se modificó durante este período con una mayor incorporación de varones a tareas domésticas y de cuidado.

Tanto los datos de la investigación previa como las nuevas entrevistas fueron transcritos en forma integral e ingresados en una Unidad Hermenéutica del programa Atlas ti, que permite analizar materiales de carácter cualitativo. El análisis se basa en las técnicas de la teoría fundamentada y del método de la comparación constante, con el objetivo de captar categorías emergentes y sistematizar su interpretación. La codificación fue realizada con un libro de códigos construido específicamente para esta investigación que contenía las principales categorías de interés, mientras se mantuvo abierta la posibilidad de registrar y crear nuevos códigos para los temas emergentes.

\section{Antecedentes sobre mediaciones parentales}

Las formas de sociabilidad y juego dentro del mundo de las infancias se han ido transformando en las últimas décadas a la luz de la masificación de las pantallas y de la difusión del uso de computadoras, tabletas y celulares. Junto con estos cambios, se han 
ido transformando las formas de parentalidad que, al acompañar los intereses de niños y niñas, han debido incorporar a las tecnologías digitales entre sus tareas. En este sentido, dentro de las prácticas de maternidad y paternidad se han vuelto centrales las actividades de mediación parental digital, esto es, las formas en las cuales los padres y las madres intermedian, gerencian y regulan los vínculos de sus hijos con las tecnologías digitales (Mascheroni, Ponte \& Jorge, 2018).

En los comienzos del desarrollo de la noción de mediación parental, la literatura se orientó hacia las actividades que los padres realizaban para regular el consumo de televisión de sus hijos (Austin, 1993; Banks \& Gupta, 1980; Nathanson, 2001, 2002; Pereira, 1998; Warren, 2005). Luego, con la aparición de nuevos medios de comunicación, con la multiplicación de las pantallas dentro de los hogares y los procesos de individualización y de miniaturización de los dispositivos, la mediación parental se desplazó de la televisión hacia todas las otras pantallas del hogar (Pasquier et al., 2012; Mostmans, 2016; Mesch, 2009 y Duek \& Moguillansky, 2020).

La literatura específica sobre mediación parental distingue entre diversos modos de intervención. Una primera clasificación propuso una escala para medir diferentes estilos, entre los que se encontraban la mediación instructiva, la restrictiva y la covisualizadora (Valkenburg, Krcmar, Peeters \& Marseille, 1999). Según Valkenburg et al. (1999) y Natansohn (1999), la mediación activa se refiere al diálogo entre padres/ madres e hijos; la mediación restrictiva involucra la fijación de reglas y el monitoreo de su cumplimiento, y la co-visualización asume que padres, madres e hijos comparten el visionado de medios.

Esta propuesta presenta dos límites: el primero es que no permite observar las modulaciones de estas prácticas de acuerdo al género, la edad, los estilos parentales y sus contextos socioeconómicos (Ponte, et al., 2019). El segundo límite, planteado por Clark (2011), es que no se ajusta bien a las nuevas formas de consumo de medios y pantallas en la era digital.

Livingstone \& Helsper (2008) realizaron una encuesta para examinar las estrategias de los padres y las madres y su correlación con una variedad de riesgos, y señalaron que el uso compartido de los dispositivos no fue efectivo para reducir los riesgos en línea. Las investigadoras concluyeron que era necesaria una nueva clasificación para comprender las estrategias de mediación parental en la contemporaneidad. Definieron cinco: 1) la mediación activa del uso de internet (para establecer diálogos sobre el uso y el contenido); 2) la mediación activa de los aspectos de seguridad de Internet (para presentar recomendaciones de uso seguro y responsable); 3) la mediación restrictiva (para establecer límites de tiempo, contenidos y actividades en línea); 4) la mediación técnica (mediante el uso de software para filtrar y/o restringir el acceso al contenido) y 5) el monitoreo (para verificar las actividades posteriores a su uso).

En todos los casos, la mediación parental es una tarea que exige de los adultos un cierto nivel de intervención en las actividades en línea de sus hijos: "los padres se sienten desafiados, especialmente porque sus hijos utilizan dispositivos móviles que son difíciles de supervisar para los padres y servicios tecnológicamente complejos que los padres pueden no entender" (Livingstone \& Byrne, 2018, p.20). Es por eso que es central visibilizar que las diferentes estrategias de mediación parental se correlacionan con varias características sociodemográficas de los padres y las madres y también con sus habilidades digitales (que se vinculan, a su vez, con sus propias trayectorias sociales) (Kirwill, Garmendia, Garitaonandia \& Martínez Fernández, 2009).

Las trayectorias con las tecnologías digitales de los adultos, junto con sus destrezas y pragmáticas de uso, acentúan o atenúan el nivel de desafío que pueden sentir madres y padres a la hora de supervisar lo que sus hijos e hijas hacen cuando se conectan. En síntesis, los adultos enfrentan desafíos y los niños y las niñas están expuestos: existe una tensión constante con respecto a lo que pueden, deben y quieren hacer con el tiempo de pantalla (Blum-Ross \& Livingstone, 2018).

En trabajos propios ya publicados (Benítez Larghi, Duek \& Moguillansky, 2017 y Duek \& Moguillansky, 2020) se ha abordado la mediación parental en Argentina y se identificaron algunos funcionamientos clave que operan como antecedentes directos de este trabajo. Un primer hallazgo 
en investigaciones previas se vinculó con la existencia de formas de mediación parental en todas las familias entrevistadas de diferentes clases sociales y regiones de la Argentina (Benítez Larghi, Duek \& Moguillansky, 2017). No se encontró ningún caso en el que no existieran formas de intervenir, limitar, mediar o negociar los tiempos en las pantallas por parte de los adultos a cargo de los niños y las niñas en cuestión. Esto no significa que las mediaciones sean similares ni que se sostengan en el tiempo, pero sí construye un dato central: la mediación parental existe y es necesario encontrar formas de organizar la relación que los más chicos tienen con las pantallas en la vida cotidiana (Duek \& Moguillansky, 2020).

Los resultados del análisis de las entrevistas realizadas coinciden en que las reglas familiares sobre las tecnologías digitales suelen ser establecidas por ambos padres, a menudo con una participación limitada de los hijos (Benítez Larghi \& Duek, 2020). Estas reglas generalmente consisten en acuerdos más o menos explícitos sobre horarios, límites de tiempo y espacios en los que los niños pueden utilizar dispositivos tecnológicos como la tableta, la televisión inteligente, la computadora, el teléfono móvil o la PlayStation (o consolas similares). Se identificó como invariante que a los niños a menudo se les permite usar la tableta o el teléfono móvil únicamente después de completar las tareas escolares.

La relación con pares apareció en los resultados como una dimensión crucial para entender la relación de los niños y las niñas con los dispositivos: las elecciones, los descartes, los juegos y el tipo de conexión que establecen siempre tiene como horizonte los vínculos actuales y los proyectados en el futuro. Clark (2011) concluye que las interacciones sobre los medios que se dan entre padres e hijos juegan un papel muy relevante en la socialización de los niños porque es a través de esas reglas, posibilidades y limitaciones que se vinculan con pares y con los contenidos a los que se exponen.

Los dispositivos disponibles, las prácticas habilitadas (o no), los tiempos de uso y de exposición, el tipo de contenidos, las configuraciones de seguridad, las excepciones y las formas de supervisión de lo hecho (revisión de historiales de búsqueda, de conversaciones, de interacciones en juegos online, entre otras variantes) son algunas de las dimensiones que emergieron como hallazgos de las investigaciones realizadas (Benítez Larghi \& Duek, 2020; Benítez Larghi, Duek \& Moguillansky, 2017). No solamente se encontraron de manera invariante formas de mediación parental, sino que se identificó una preocupación de los adultos por comprender los usos y las necesidades de sus hijos e hijas en su tiempo de conexión.

Un segundo hallazgo de las investigaciones previas es que no prevalece una forma única de mediación parental por sobre las otras, sino que las cinco variantes suelen estar presentes con diferentes intensidades y gradaciones. Desde la mediación activa del uso hasta el monitoreo y el control, pasando por la seguridad, las restricciones y las mediaciones técnicas, las modalidades coexisten dentro de los hogares de manera simultánea con diferente gradación a lo largo del tiempo y de las dinámicas familiares. Esta conclusión deja en evidencia que la relación que los adultos establecen con las modalidades y con los tiempos de uso de los dispositivos por parte de sus niños y niñas está atravesada por variables diversas que se explican solamente en los contextos familiares y vinculares en los que se insertan.

El tercer hallazgo se vincula con el género: se identificó que la mediación parental estaba a cargo de las mujeres más que de los varones. El seguimiento, las negociaciones, las conversaciones y las explicaciones se encontraban en el terreno de las madres. Tanto los niños y las niñas como los adultos entrevistados identificaron como natural esta distribución de tareas respecto de las actividades de los más chicos con relación a las pantallas (Duek \& Moguillansky, 2020). La percepción de una supuesta naturalidad se vincula con el efecto de la reproducción de un funcionamiento social hegemónico que no se cuestiona (Faur, 2012) y que se repite.

Las madres entrevistadas mencionaron pasar más tiempo con sus hijos e hijas que sus maridos o exmaridos y la mediación es una tarea que queda de su lado. Esta división generalizada del trabajo involucrada en la mediación parental está vinculada con un sentido 
más amplio y construido de estereotipos de género y de ideologías (Livingstone, Talves \& Kalmus, 2015). A lo largo de la historia se han establecido asociaciones entre el cuidado y el género, ubicando primordialmente a las mujeres como sujetos a cargo de la cotidianidad de los niños y las niñas.

La relación entre la maternidad como construcción social y las tareas de cuidado fue analizada por Faur (2012), quien afirma que la presencia de las madres en la crianza de sus hijos es mayor que la de los padres. Y es esa presencia la que constituye, a la vez, la síntesis de un mandato social ("Las madres deben ocuparse de los hijos") y de la asignación de tareas ("Esos temas los saben las madres"). En este sentido, no es sorprendente que las excepciones identificadas al hallazgo mencionado se relacionan con problemas vinculares, conflictos con pares o con las configuraciones, que eran los momentos en los que se convocaba puntualmente a los padres para que intervinieran y resolvieran la situación planteada (Moguillansky \& Duek, 2020).

Estos tres hallazgos son antecedentes directos de los resultados que se presentarán a continuación sobre mediación parental en pandemia y las modalidades y variantes que asumieron las negociaciones en torno a los usos, las reglas, los límites y los tiempos de los dispositivos electrónicos.

\section{Resultados: la mediación parental en pandemia}

\section{Dispositivos y mediaciones}

La pandemia y las cuarentenas extendidas trastocaron las rutinas familiares, las modalidades de educación, el entretenimiento y la sociabilidad (Koury \& Moguillansky, 2021). En las vidas de los niños y las niñas, según Aliano (2021), se produjeron tres cambios simultáneos: a) se interrumpió la interacción presencial entre pares que era, hasta entonces, la forma dominante de sus vínculos de amistad; b) se volvió imperativo organizar el cuidado en el hogar por parte de familiares; y c) las escuelas comenzaron a proponer rutinas educativas a distancia, creando ocasiones de uso de pantallas novedosas. Estos tres cambios tuvieron un carácter abrupto (Aliano, 2021) y confluyeron en la generación del escenario para un cambio en las modalidades de la mediación parental con respecto a las tecnologías.

Durante la pandemia se observó, en muchos casos, un uso más intensivo, frecuente y diversificado de las pantallas por parte de los niños y las niñas; esto fue posible debido a que se flexibilizaron las reglas y los límites que los padres establecían con sus hijos. Ante la necesidad de tener entretenidos y ocupados a los niños, se habilitó un mayor tiempo de uso de pantallas y un menor control de los contenidos o las aplicaciones utilizados, recurriendo así a los dispositivos como una suerte de niñera virtual (en línea con investigaciones previas que definieron a la televisión como un chupete electrónico, como Maccoby, 1951 y, más recientemente, Diefenbach \& Borrmann, 2019, que usaron el mismo concepto para definir la relación de los adolescentes con sus celulares).

Sin embargo, en el contexto particular de la pandemia, estos cambios adquieren modulaciones específicas según las condiciones de clase de las familias, el tipo de ocupación de los padres y el tipo de escolaridad (simple o extendida) a la que asisten los hijos.

En las entrevistas realizadas se verifica que en las familias de clase media con hijos en edad escolar se relajaron las reglas de uso de celulares, tabletas, consolas de videojuegos y computadoras durante los primeros meses de la pandemia. La percepción dominante entre los padres y las madres entrevistados es que en los primeros meses de la cuarentena en sus hogares se vivió cierto "descontrol", “caos" y "desorden” con relación a sus hijos y a los usos de aparatos electrónicos.

Al principio fue más desordenado todo en casa, después nos fuimos armando una rutina, pero muchas veces era tierra de nadie para mi hijo, por ejemplo a la mañana yo tenía que trabajar desde casa y él estaba prendido a la tele, nadie le daba bola. Cambiaron mucho las reglas, empezó a usar más la tablet y la computadora, que antes casi no le dejábamos. (Participante 1 , madre de un niño de 6 años, escuela privada) 
En muchos hogares, durante la pandemia, se adquirieron nuevos dispositivos y/o se reorganizó el uso de los aparatos existentes, para lograr que cada niño o niña tuviera la posibilidad de utilizar un celular o una tableta para las actividades escolares o para el tiempo libre. Los hogares de las familias entrevistadas de clase media contaban, en promedio, antes de la pandemia, con un celular por cada adulto o adolescente, una o más computadoras y algún otro dispositivo lúdico (consolas, tabletas). Aún con esta abundancia tecnológica, no siempre resultó suficiente durante la etapa de aislamiento de la pandemia, puesto que todos los miembros del hogar requerían el uso de dispositivos digitales y, en reiteradas ocasiones, de manera simultánea.

Así, por ejemplo, en la casa de una de las madres entrevistadas, hicieron tanto compras como cambios del uso de los dispositivos:

Justo antes de la pandemia habíamos comprado una Playstation. Ya en la pandemia compramos celulares para nosotros y además hubo una redistribución dentro de casa: dos celulares que estaban viejos pasaron a manos de los chicos, que hasta ese momento no tenían ningún dispositivo. Había una tableta de uso familiar que pasaron a usar más ellos. Compramos también un escritorio para mí, armamos uno para mi hijo más grande y le compramos auriculares con micrófono. (Participante 2, madre de dos niños de 8 y de 5 años, escuela pública)

En varios de los testimonios recogidos, los padres y las madres señalan que decidieron otorgarles un celular a sus hijos (en algunos casos con chip y en otros no, lo que los convierte, prácticamente, en una tableta) para usarlo como propio, aunque previamente habían planeado que esto ocurriría a una edad más avanzada. La motivación fue lograr que sus hijos pudieran tener contacto con sus pares, a través de las redes sociales.

Durante la cuarentena, los juegos aparecieron como prácticas significativas sostenidas con pares mediante diferentes dispositivos. Una modalidad de juego a distancia que relatan varios entrevistados es, precisamente, el juego en red acompañado en simultáneo por una llamada o videollamada, para comentar las jugadas o concertar estrategias conjuntas. Los juegos más mencio- nados fueron la plataforma interactiva Roblox, el Brawl Stars y Among Us.

En algunas familias, también se asignó o se regaló a los chicos y las chicas una computadora personal, para seguir las clases de la escuela. No obstante, estos cambios y esta caracterización acerca de los mayores permisos y la posesión de dispositivos propios requieren matices de acuerdo a las condiciones socioeconómicas de las familias, puesto que responden más bien a la situación de niños y niñas de clase media en entornos urbanos.

La situación fue diferente en los hogares de sectores populares, donde muchas veces se disponía de un único dispositivo para realizar las diversas actividades educativas, de sociabilidad y de entretenimiento. En las familias de menores recursos, la pandemia fue un desafío que se atravesó regulando más estrictamente los usos de los aparatos electrónicos por su escasez más que por preocupaciones de sobreexposición. Así, por ejemplo, una informante da cuenta de su experiencia:

En mi casa tenemos solo mi celular, no hay computadora ni nada. Sí cambiaron las cosas con las reglas de uso sobre el celular, porque antes de pronto era un tiempito quizás para que los chicos jugaran o de pronto se distrajeran en algo, pero en este momento fue algo para la enseńanza, el uso del celular se volvió algo didáctico. No compramos ningún celular, nos manejamos toda la pandemia con el mío, que dentro de sus limitaciones pudimos resolver bastante. (Participante 3, madre de un niño de 8 años y de una niña de 11 , escuela pública, CABA)

La administración y la gestión del tiempo de uso del celular fue una tarea demandante porque confluían en un único dispositivo la necesidad de uso escolar y laboral pero, también, los deseos de conexión con pares y de entretenimiento mediante juegos descargados en ese teléfono.

\section{Conexión, nuevas destrezas y control}

A pesar del contraste entre las familias de clase media con abundancia de dispositivos y conectividad, por un lado, y las familias de sectores populares con límites en ambas dimensiones, por otro, también 
aparecen aspectos en común. La perspectiva de los padres y de las madres sobre las experiencias de sus hijos con las tecnologías durante la pandemia destaca los aprendizajes de destrezas digitales nuevas, tales como participar de una videollamada, enviar correos electrónicos, adjuntar archivos, utilizar procesadores de texto, entre tantas otras.

Veo que los chicos ganaron muchas destrezas que obviamente no las van a perder, por ejemplo, aprendió a usar el Zoom y el Meet, a adjuntar archivos, mandar mails, digitalizar imágenes. Todo eso, las llamadas en grupo con los amigos, formas nuevas de jugar, son cambios que creo que van a quedar. (Participante 4, madre de un niño de 8 años, escuela pública)

Otro aspecto relevante por destacar del análisis de las entrevistas es que se incrementó notoriamente la posibilidad de los padres y las madres de observar de cerca e, inclusive, de compartir algunas de las prácticas lúdicas y educativas de sus hijos con tecnologías digitales. Así, las formas de mediación parental que suponen la participación activa del adulto se vieron intensificadas cuanti y cualitativamente: padres o madres que jugaron con sus hijos, que los vieron como estudiantes, como amigos de otras personas, que compartieron más comidas y espacios que antes y que, en algunos casos, lograron comprender significados y prácticas de las vidas de sus hijos e hijas a los que, según los testimonios, no hubieran tenido acceso en la vida previa a la pandemia (o, al menos, hubiera sido más difícil su reconstrucción).

Ahora bien, un aspecto relevante que surge del análisis de las entrevistas es que, tras un primer momento de flexibilización de las reglas y de un uso más intensivo de las pantallas por parte de los niños o las niñas, que se percibe y recuerda como etapa de "descontrol", los adultos comienzan a volver a establecer límites, a crear rutinas y delimitar momentos para apagar los dispositivos.

En ese sentido, una informante relató que en su hogar antes de la pandemia no tenían reglas fijas para el uso de dispositivos, pero controlaban la cantidad de tiempo que su hija de 8 ańos pasaba frente a pantallas. Al comenzar el aislamiento y no contar con otras opciones para entretener a su hija, flexibilizaron esos límites; la informante relató que comenzó a prestarle más seguido su propio celular y le permitió abrir cuentas en aplicaciones como Tik Tok o Roblox. Sin embargo, luego percibieron que era problemático y dieron marcha atrás:

Con la pandemia cambiaron las reglas, sí, en algunos momentos difíciles la pantalla hizo de niñera para mi hija; estaba con Roblox y TikTok, yo se la abrí para que pudiera editar videos, pero se terminó desvirtuando, era un vicio, estaba como una persona adicta. Eso se lo fuimos prohibiendo y finalmente se lo sacamos porque era incontrolable. (Participante 4, madre de una niña de 8 años, escuela privada)

Siguiendo el relato de la madre, resulta claro que, luego de unos meses, comenzaron a proponer de nuevo algunos límites al uso de celulares y tabletas, a observar qué tipo de contenidos eran apropiados para ella y orientar sus actividades online. En los hogares y en las interacciones familiares se vuelve necesaria la "producción de un nuevo orden doméstico” (Aliano, 2021, p. 32) a través de la segmentación, el ordenamiento y la secuenciación de las prácticas de la vida cotidiana, es decir, de la gestación de nuevas rutinas.

Este nuevo orden doméstico que se fue creando luego de varios meses de pandemia fue de la mano con otros cambios en el contexto, tales como la relajación de los controles de circulación, la habilitación del uso de parques y plazas, y las propuestas de actividades educativas a distancia por parte de las escuelas. Según los entrevistados, hacia mediados del ańo, pudieron volver a contar con niñeras o relevos en el hogar, los paseos al aire libre se volvieron más frecuentes y las escuelas ofrecieron, en la mayoría de los casos, algunos encuentros sincrónicos por videollamada.

En la segunda mitad del ańo cuando se reorganizó un poco la rutina escolar y empezó a venir la niñera, regulamos un poco mejor el uso de los celulares, ellos empezaron a ir a la plaza, tenían las actividades de la escuela y talleres a la tarde, así que no le quedaba tanto tiempo para jugar online. (Participante 2, madre de dos niños de 8 y 5 años, escuela pública) 
La dimensión clave que modula la intensidad del uso de pantallas es el tiempo: cuando la pandemia y el aislamiento suspendieron las prácticas habituales de los niños y las niñas, las infancias tuvieron mucho tiempo libre y los adultos relajaron los límites que previamente sostenían para conseguir llenar ese tiempo. Cuando el aislamiento se fue tornando menos estricto y aparecieron otras actividades habilitadas, las familias volvieron a regular el uso de pantallas y las rutinas de los niños y las niñas se reorganizaron.

\section{Discusión y conclusiones}

Las estrategias de mediación parental de las tecnologías se modificaron durante la pandemia. En este trabajo se presentaron resultados sobre los cambios en las formas de intervención de los padres sobre los usos de tecnologías por parte de niños y niñas, en contraste con las formas prevalecientes de mediación parental antes de la pandemia. A través de entrevistas estructuradas, se reconstruyó la perspectiva de los adultos acerca de sus estrategias de regulación y monitoreo del uso de tecnologías digitales por parte de sus hijos. Así, se observó que se multiplicó el tiempo con pantallas en diversos dispositivos, que surgieron nuevas destrezas tecnológicas y que, con el tiempo, en las familias se crearon nuevas rutinas y formas de regulación de las actividades online de los niños.

Los hallazgos de esta investigación con respecto a los cambios de las prácticas de mediación parental pueden ser discutidos recurriendo a la tipología de Livingstone \& Helsper (2008). Durante la pandemia, se incrementaron las prácticas de mediación activa del uso de Internet, es decir, los diálogos de los padres con sus hijos acerca de sus actividades online, como resultado de una mayor disponibilidad de tiempo compartido en familia y al aumento del tiempo compartido en los hogares.

A su vez, se intensificó notoriamente la mediación activa sobre la seguridad de Internet, tarea a la cual se incorporaron los docentes y las instituciones educativas, que se vieron obligadas a realizar sus actividades online y tuvieron que enfrentar diversos peligros que suponía esta situación (robo de claves, usurpación de identidad y diversos problemas vinculados con los riesgos globalmente conocidos y relevados de la conectividad).

Los resultados confirman la conclusión de Livingstone \& Byrne (2018), quienes sostienen que el rol de los adultos en la mediación parental supone un desafío porque los dispositivos móviles que usan sus hijos e hijas son difíciles de supervisar. La pandemia mostró que, frente a la flexibilización de algunas reglas por falta de alternativas de sociabilidad presencial y por la superposición de actividades de miembros del hogar, la mediación parental se volvió aún más desafiante: no solo se trata de negociar, intervenir y construir reglas, sino de sostenerlas sin relevos, sin ayuda y con casi nulas alternativas y opciones fuera del hogar.

En este sentido, no es sorprendente que aparezcan de manera invariante nuevas reglas y mediaciones al mismo tiempo que se configura una (cierta) rutina en el segundo semestre. La rutina apareció en las entrevistas como algo "perdido" por la falta de presencialidad, pero ni bien las escuelas mostraron un nivel de organización en la virtualidad y/o en las alternativas presentadas a las familias (no todas al mismo tiempo ni con la misma eficacia), se reconoce un nuevo punto de equilibrio que se funda en estrategias de mediación y de regulación de los tiempos de conexión de los niños y de las niñas.

Si un niño o una niña recibió un nuevo dispositivo, es muy difícil pensar, incluso con el retorno de las clases presenciales, en quitárselo de manera definitiva. El nuevo punto de equilibrio supondría, en este caso, contemplar ese nuevo dispositivo como elemento "a regular". La mayoría de los adultos coincide en que hay un cierto "terreno ganado" por los niños y las niñas con respecto a los usos de pantallas. Así como se reconocen las nuevas destrezas que incorporaron los más chicos como ganancias y aprendizajes centrales de la pandemia, los adultos entrevistados coinciden en que toda discusión sobre los usos de dispositivos electrónicos tiene un nuevo punto de partida que incluye más permisos y más horas de conectividad semanal que en la vida previa a la pandemia.

No se detectaron cambios con respecto a la mediación técnica, que referiría al uso de aplicaciones 
especiales para controlar el tiempo de uso o filtrar algunas aplicaciones o algunos contenidos problemáticos. Este sería un aspecto para continuar explorando en futuras investigaciones y para indagar si su continuidad no alterada se vincula con la falta de tiempo por el aumento de demandas que supuso la pandemia en los hogares o si se vincula, como hipótesis, con la falta de destrezas específicas para llevarla a cabo.

Con relación a las prácticas de monitoreo que antes de la pandemia consistían en verificar durante o después de las actividades online los recorridos de los niños o las niñas, estas se volvieron constantes durante la pandemia, puesto que las clases virtuales requerían de la presencia de algún adulto para verificar si la conexión funcionaba bien, si el nombre era el correcto, si estaban prestando atención y comprendiendo las consignas, entre otras tareas.

Con respecto al género como configurador de las mediaciones parentales, antes se señaló que en la etapa previa a la pandemia estas prácticas estaban más a cargo de las mujeres que de los varones. Con la pandemia y el aislamiento se produjeron algunas transformaciones en la distribución de roles y tareas dentro del hogar (que no solo impactaron en las mediaciones de las tecnologías). La mayor presencia de los varones en el hogar, como señalan Canevaro \& Castilla (2021), no significó que se involucrasen de forma decisiva en las tareas de cuidado de niños y niñas, a diferencia de lo que sostenía una de las hipótesis de investigación.

En las entrevistas realizadas, se observa que el patrón de género se mantiene a grandes rasgos: las madres se ocupan más de acompañar la educación a distancia, de monitorear la conexión de sus hijos y de conversar con ellos sobre sus actividades online. Cuando en el hogar hay dos niños o niñas, por lo general las madres se ocupan del más pequeño y los padres del más grande. Los padres se ocupan de las salidas al mundo exterior no-doméstico, aparecen cuando hay algún problema específico, cuando las madres se ven sobrepasadas o, en algunos casos, si hay una división explícita de tareas en la que ellos asumen alguna parte del cuidado y/o la mediación parental.

En síntesis, en este trabajo se procuró revisar las discusiones sobre las prácticas de mediación parental del uso de tecnologías de niños y niñas, comparando las rutinas previas a la pandemia con las formas de intervención que se fueron creando durante el aislamiento en sus diferentes etapas. Se ha mostrado que el comienzo de la pandemia supuso cambios abruptos en la vida doméstica, laboral y cotidiana y que las prácticas de mediación parental se vieron afectadas, con un relajamiento de los límites de uso para niños y niñas.

Con el paso del tiempo y la extensión del aislamiento, se crearon nuevas rutinas y se distribuyó el uso del tiempo volviendo a limitar y regular la relación con las pantallas. De esta manera, el trabajo propone un aporte para comprender mejor las consecuencias de la pandemia en las formas de vinculación entre adultos, niños y pantallas, comprendiendo que la mediación parental está necesariamente atravesada por el contexto dinámico, cambiante y desafiante en el que se desarrolla.

Los efectos de la pandemia en las modificaciones de las negociaciones, de las reglas y de los límites de la relación de los más chicos con las pantallas han sido explorados aquí y las nuevas modalidades de mediación en la salida de la pandemia hacia la presencialidad escolar y laboral serán, seguramente, temas para futuras investigaciones.

\section{Referencias}

Aliano, N. (2021). La reinvención de lo cotidiano en tiempos de pandemia. Aislamiento, usos de la casa y estrategias de ordenamiento de las rutinas en hogares de clase media en Argentina. Revista Brasileña de Sociología de las Emociones, 20(58), 21-34.

Austin, E. W. (1993). Exploring the effects of active parental mediation of television content. Journal ofBroadcastingandElectronicMedia,37,147-158. https://doi.org/10.1080/08838159309364212

Banks, S., \& Gupta, R. (1980). Television as a dependent variable, for a change. Journal of Consumer Research, 7(3), 327-330. https://www.jstor.org/ stable/2489017?seq=1

Baratta, M. V. (2021). No esenciales. La infancia sacrificada. Libros del Zorzal. 
Becerra, M. (2021). Accesos tic 2000-2020 en Argentina: ¿20 años no es nada? Conectividad y brechas en telecomunicaciones, internet y tv paga en el siglo XXI, Informe preliminar sobre conectividad en Argentina 2000-2020. https://martinbecerra. wordpress.com/2021/06/16/accesos-tic-20002020-en-argentina-20-anos-no-es-nada/

Benítez Larghi, S., \& Duek, C. (2020). Tecnologías, género y socialización: dos tesis para pensar las infancias, Revista Papeles de trabajo, (24), 39-54.

Benítez Larghi, S., Duek. C. \& Moguillansky, M. (2017). Infancias y tecnologías en Argentina: interacciones y vínculos intergeneracionales, Revista Nómadas. Tramas contemporáneas en comunicación y educación, 1(49), 79-109.

Blum-Ross, A., \& Livingstone, S. (2018). The Trouble with "Screen Time" Rules". In Mascheroni, G., Ponte, C. \& Jorge, A. (eds.), Digital Parenting. The Challenges for Families in the Digital Age (pp.179-188), The International Clearinghouse on Children, Youth and Media, at Nordicom University of Gothenburg. https:// www.nordicom.gu.se/sv/system/tdf/publikationer-hela-pdf/yearbook_2018_digital_parenting. pdf?file $=1 \&$ type $=$ node $\&$ id $=39884 \&$ force $=0$

Canevaro, S. \& Castilla. M.V. (2021). Masculinidad, intimidad y cuidados: ¿nuevas reconfiguraciones en la pandemia? Revista Brasileña de Sociología de las Emociones, 20(58), 97-113.

Clark, L. (2011). Parental mediation theory for the digital age. Communication theory, 21(4), 323-343. https://doi.org/10.1111/j.1468-2885. 2011.01391.x

Duek, C. (2021). La educación virtual en Argentina: límites y posibilidades de la educación en el contexto de la pandemia 2020. Revista Brasileña de Sociología de las Emociones, 20(58), 127-138.

Duek, C., \& Moguillansky, M. (2020). Children, digital screens and family: parental mediation practices and gender, Comunicacao e sociedade, 1(37), 65-80, Centro de estudos de comunicacao e sociedade, Universidade De Minho. https:// doi.org/10.17231/comsoc.37(2020).2407
Diefenbach, S. \& Borrmann, K. (2019). The Smartphone as a Pacifier and its Consequences: Young adults' smartphone usage in moments of solitude and correlations to self-reflection, Proceedings of the 2019 CHI Conference on Human Factors in Computing Systems (CHI '19). Association for Computing Machinery, New York, NY, EE. UU. https://doi.org/10.1145/3290605.3300536

Faur, L. (2012). El cuidado infantil en el siglo XXI. Siglo XXI.

Giudici, S. (2018). Informe de gestión ENACOM, Secretaría de Modernización, Presidencia de la Nación. https://www.enacom.gob.ar/informes-de-gestion_p3218

INDEC. (2017). Documentos. Censo nacional, Argentina.

Kirwill, L., Garmendia, M., Garitaonandia, C., \& Martínez Fernández, G. (2009). Parental mediation, S. Livingstone and L. Haddon (Eds.), Kids online: Opportunities and risks for children. Policy Press, 199-215.

Koury, Mauro \& Moguillansky, Marina. (2021). Presentación del dossier "Vida cotidiana, emociones y situaciones límite: vivir en un contexto de pandemia, Revista Brasileña de Sociología de las Emociones, 20(58), 9-14.

Livingstone, S., \& Byrne, J. (2018). Parenting in the Digital Age. The Challenges of Parental Responsibility in Comparative Perspective, Mascheroni, G., Ponte, C., \& Jorge, A. (Eds.), Digital Parenting. The Challenges for Families in the Digital Age, 19-30. The International Clearinghouse on Children, Youth and Media, at Nordicom University of Gothenburg. https://www.nordicom. gu.se/sv/system/tdf/publikationer-hela-pdf/yearbook_2018_digital_parenting.pdf?file $=1 \&$ type $=$ node $\&$ id $=39884 \&$ force $=0$

Livingstone, S., Talves, K., \& Kalmus, V. (2014). Girls' and boys' experiences of online risk and safety. In C. Carter; L. Steiner \& L. McLaughlin (Eds.), The Routledge companion to media and gender, 190-200. Routledge. 
Livingstone, S. \& Helsper, E. (2008). Parental mediation of children's Internet use. Journal of broadcasting and electronic media, 52(4), 581-599. https:// doi.org/10.1080/08838150802437396

Maccoby, E. (1951). Television: Its Impact on School Children, Public Opinion Quarterly, 15(3), 421-444. https://doi.org/10.1086/266328

Mascheroni, G., Ponte, C., \& Jorge, A. (2018). Introduction in Mascheroni, G., Ponte, C., \& Jorge, A. (Eds.), Digital Parenting. The Challenges for Families in the Digital Age, pp. 9-18. The International Clearinghouse on Children, Youth and Media, at Nordicom University of Gothenburg. https://www.nordicom.gu.se/sv/system/ tdf/publikationer-hela-pdf/yearbook_2018_ digital_parenting.pdf?file $=1 \&$ type $=$ node $\&$ i$\mathrm{d}=39884 \&$ force $=0$

Mesch, G. (2009). Parental mediation, online activities, and cyberbullying. Cyberpsychology \& behavior: the impact of the Internet, multimedia and virtual reality on behavior and society, 12(4), 387-93. https://www.doi.org/10.1089/ cpb.2009.0068

Mostmans, L. (2016). Internet mediation and the family gap: explorative ethnographic interviews in new family forms in Belgium. Journal of Children and Media, 10(4), 481-496. https://www. doi.org/10.1080/17482798.2016.1222298.

Nathanson, A. (1999). Identifying and explaining the relationship between parental mediation and children's aggression. Communication research, 26(2), 124-143. https://doi.org/10.1177/0093 65099026002002

Nathanson, A. (2001). Parent and Child Perspectives on the Presence and Meaning of Parental Television Mediation. Journal of Broadcasting \& Electronic Media, 45(2), 201-220, https://doi. org/10.1207/s15506878jobem4502_1

Nathanson, A. (2002). The unintended effects of parental mediation of television on adolescents. Mediapsychology, 4, 207-230. https://doi. org/10.1207/S1532785XMEP0403_01
Pasquier, D., Simóes, J.A., \& Kredens, E. (2012). Agents of mediation and sources of safety awareness: a comparative overview, In Livingstone, S., Haddon, L., \& Görzig, A. (Eds). Children, risk and safety on the internet. Research and policy challenges in comparative perspective. Polity Press (pp. 219-230).

Pereira, S. (1998). A televisão na família. Processos de mediação com crianças em idade pré-escolar. Instituto de estudos da criança. http://repositorium. sdum.uminho.pt/bitstream/1822/4265/1/ TVnaFam\%c3\%adlia.pdf

Ponte, C., Simoes, J., Batista, S. \& Castro, T. (2019). Implicados, intermitentes, desengajados? Estilos de mediação de pais de crianças de 3-8 anos que usam a internet. Sociologia, problemas e práticas, (91), 39-58. http://journals.openedition.org/ spp/6442

SINCA [Sistema de Información Cultural de la Argentina]. (2017). Los jóvenes y los consumos culturales. Informe de investigación sobre los consumos culturales de los jóvenes en la Argentina, basado en los resultados de la Encuesta Nacional de Consumos Culturales, edición 2017. Ministerio de Cultura de la Nación. https://www.cultura. gob.ar/media/uploads/jovenes_y_consumos_ culturales_1.pdf

Valkenburg, P., Krcmar, M., Peters, A., \& Marseille, N. (1999). Developing a scale to assess three different styles of television mediation: 'Instructive mediation', 'restrictive mediation', and 'social coviewing'. Journal of broadcasting and electronic media, 43(2), 52-66.

Warren, R. (2005). Parental mediation of children's television viewing in low income families. Journal of Communication, 55, 847-863. https://doi. org/10.1111/j.1460-2466.2005.tb03026.x 